\title{
SAR Image Change Detection Based on Multi-scale Feature Extraction
}

\author{
Xiaoqian Yuan \\ Chengdu University \\ of Information \\ Technology \\ Chengdu, China
}

\author{
Chao Chen \\ Chengdu University \\ of Information \\ Technology \\ Chengdu, China
}

\author{
Shan Tian \\ Chengdu University \\ of Information \\ Technology \\ Chengdu, China
}

\author{
Jiandan Zhong \\ Chengdu University \\ of Information \\ Technology \\ Chengdu, China
}

\begin{abstract}
In order to improve the contrast of the difference image and reduce the interference of the speckle noise in the synthetic aperture radar (SAR) image, this paper proposes a SAR image change detection algorithm based on multi-scale feature extraction. In this paper, a kernel matrix with weights is used to extract features of two original images, and then the logarithmic ratio method is used to obtain the difference images of two images, and the change area of the images are extracted. Then, the different sizes of kernel matrix are used to extract the abstract features of different scales of the difference image. This operation can make the difference image have a higher contrast. Finally, the cumulative weighted average is obtained to obtain the final difference image, which can further suppress the speckle noise in the image.

Keywords: SAR image change detection; fuzzy c-means clustering; multi-scale feature
\end{abstract}

\section{INTRODUCTION}

SAR image change detection is the process of comparing the difference between two or more images in the same area at different times and extracting the change area. SAR change detection has been widely used in many fields such as military detection, land desertification detection, vegetation change analysis, geological disaster assessment, and urban change research. Traditional change detection algorithms can be divided into supervised change and unsupervised change detection algorithms according to whether the prior knowledge is required. Due to the limited conditions and high cost of obtaining prior knowledge in SAR image change detection, unsupervised change detection is more popular among scholars.

SAR image change detection mainly includes three steps: image preprocessing, construction of difference image and extraction of change information. Constructing a highperformance difference image is a key step in unsupervised change detection. Common difference operations include difference method and ratio method. Ratio method mainly includes log-ratio (LR) method and mean value ratio (Meanratio, MR) method. The speckle noise in the SAR image can be converted into additive noise by using LR method, and the variation range of the logarithmic ratio image result can be compressed. MR method uses information of neighborhood pixels and can appropriately suppresses the background information of the image. On this basis, Gong et al. proposed the Neighborhood-ratio (NR) algorithm, which uses the heterogeneity measurement coefficient $\partial$ to effectively combine the mean method and the ratio method. Since then, many scholars have adopted a variety of differential operations. For example, Gong et al. used wavelet transform to fuse mean ratio and log ratio difference images, and Jiang et al. used Fourier transform to fuse difference image and log ratio image. Zhou et al. used a linear combination method to combine the mean ratio difference image and the difference method difference image. The above algorithms appropriately suppress the speckle noise in the difference image, but the generated difference image has low contrast and more speckle noise, and the outline of the image is not clear enough, which is not conducive to subsequent classification. Because the difference image generated by the traditional algorithm has certain limitations, the quality of the difference image not only affects the accuracy of subsequent classification, but also affects the detection effect of the detection algorithm as a whole. This paper uses a weighted pooling kernel matrix to extract features from two original images by simulating convolution and pooling operations. Because the features extracted at a single scale are not comprehensive enough, this paper accumulates weighted pooling and merges the image features extracted by different window sizes. The final difference image is formed by one, and a method based on multi-scale features is proposed to construct the difference image.

\section{SAR IMAGE CHANGE DETECTION BASED ON MULTI-SCALE FEATURE EXTRACTION}

\subsection{Difference Image Based on Multi-Scale Feature Extraction}

Affected by the feature extraction of the deep neural network, the average pooling operation can effectively suppress the noise, resulting in the loss of a large amount of detailed information. Maximum pooling operation, it can effectively enhance the image characteristics, while being very sensitive to noise. Traditional difference image operators have certain defects when constructing difference images. Therefore, this paper proposes a weighted pooling method based on local window distance metric to generate difference image. This method can effectively extract the original image features, obtain a clearer image contour of the changed area, better retain the edge information of the image, and better suppress the noise interference of unchanged area. The changed area and the unchanged area have a higher contrast, which is beneficial to subsequent classification. 
Assuming that the local window size is $k \times k, k$ is an odd number, and the weighted pooling kernel matrix is $W^{k}$, the formula 2-1 gives its matrix form as follows:

$$
W^{k}=\left(\begin{array}{cccc}
w_{11} & w_{12} & \cdots & w_{1 k} \\
w_{21} & w_{22} & \cdots & w_{2 k} \\
\vdots & \vdots & \ddots & \vdots \\
w_{k 1} & w_{k 2} & \cdots & w_{k k}
\end{array}\right)
$$

The value of each element in the matrix is determined by the current position and the position of the center of the matrix, which can be calculated by formula 2-2. In particular, the value of the element in the center of the matrix is initialized as $w_{(k+1) / 2,(k+1) / 2}=2 / k^{2}$.

$$
w_{i j}=\frac{1}{k^{2} \sqrt{\left(\frac{k+1}{2}-i\right)^{2}+\left(\frac{k+1}{2}-j\right)^{2}}}
$$

Given two multi-temporal SAR images $I_{1}$ and $I_{2}$, using the same kernel matrix to convolve the two SAR images can obtain two feature images, $I_{1}^{w p}(k)=I_{1} * W^{k}, I_{2}^{w p}(k)=I_{2} * W^{k}$, * denotes the two-dimensional convolution operation. In the same way, the value of each pixel after the weighted pooling operation of the image and the kernel matrix can be calculated by formula 2-3. Then use equation 2-4 to find the logarithmic ratio difference image after weighted pooling.

$$
\begin{gathered}
I_{\mathrm{nm}}^{w p}=\frac{1}{k^{2}} \times \sum_{i=1}^{k} \sum_{j=1}^{k} w_{i j} \times I_{n+i-(k+1) / 2, m+j-(k+1) / 2} \\
I_{d}=\left|\log \left(I_{2}^{w p} / I_{1}^{w p}\right)\right|
\end{gathered}
$$

The anti-noise performance of the logarithmic ratio operator is weak, and there is still a certain degree of speckle noise in the obtained difference image. Based on the sparse distribution of noise outliers and the clustered distribution of real changes in the log ratio image, we apply kernel matrices with different local window sizes to the difference image to obtain features of different scales of the image. Perform the above operations on each pixel in the difference image, and then accumulate and weight-average images with different window sizes $\mathrm{T}$ times to obtain the difference image. Its calculation formula is given as follows:

$$
\begin{aligned}
I_{D D I} & =\frac{1}{T} \sum_{\mathrm{t}=1}^{T} \frac{I_{\mathrm{d}}^{w p}(2 t-1)}{\bar{w}} \\
\bar{w} & =\frac{1}{(2 t-1)^{2}} \sum_{i=1}^{2 t-1} \sum_{j=1}^{2 t-1} w_{i j}
\end{aligned}
$$

Where $I_{\mathrm{d}}^{w p}(2 t-1)$ represents the weighted pooled image with the size of the local window is $k=2 t-1$. The difference image is obtained by the cumulative summation of the above formula, and the final difference image is obtained by normalization.

\subsection{Modified Fuzzy C-means Clustering Algorithm}

Because the traditional FCM algorithm clusters each sample pixel as a separate sample pixel, and does not consider the neighborhood factor of the pixel when segmenting the image, so it is more sensitive to noise. In view of this, Ahmed et al. proposed a modified fuzzy C-Means (FCM_S) algorithm. The FCM_S algorithm considers the spatial factors of pixels on the basis of the FCM algorithm, which improves the accuracy of image segmentation to a certain extent. By introducing the neighborhood regular term, the classification of the center pixel is affected by the neighborhood pixels, and the modified objective function is:

$$
\begin{aligned}
J_{m}(U, V) & =\sum_{i=1}^{n} \sum_{k=1}^{c} u_{i k}^{m}\left\|x_{i}-v_{k}\right\|^{2} \\
& +\frac{\alpha}{N_{R}} \sum_{i=1}^{n} \sum_{k=1}^{c} u_{i k}^{m} \sum_{x_{r} \in N_{i}}\left\|x_{r}-v_{k}\right\|^{2}
\end{aligned}
$$

in the above formula, represents the neighborhood set of pixel $x_{i}$, and $N_{R}$ represents the number of pixels in the neighborhood. $\alpha$ represents the adjustment parameter of the neighborhood item to the center pixel. According to the objective function of the FCM_S algorithm combined with the Lagrange multiplier method, the membership function and the iterative formula of the clustering center can be derived as follows:

$$
\begin{gathered}
u_{i k}=\frac{\left(\left\|x_{i}-v_{k}\right\|^{2}+\frac{\alpha}{N_{R}} \sum_{r \in N_{i}}\left\|x_{r}-v_{j}\right\|^{2}\right)^{-1 /(m-1)}}{\sum_{j=1}^{c}\left(\left\|x_{i}-v_{j}\right\|^{2}+\frac{\alpha}{N_{R}} \sum_{r \in N_{\mathrm{i}}}\left\|x_{r}-v_{j}\right\|^{2}\right)^{-1 /(m-1)}} \\
v_{k}=\frac{\sum_{i=1}^{n} u_{i k}^{m} x_{i}+\sum_{i=1}^{n} \frac{\alpha}{N_{R}} \sum_{r \in N_{i}} x_{r}}{(1+\alpha) \sum_{i=1}^{n} u_{i k}^{m}}
\end{gathered}
$$

\section{EVALUATION CRITERIA}

This paper uses the following evaluation criteria to objectively evaluate the results of change detection:

(1) False negative (FN) number: the number of pixels that actually changed that were finally detected as unchanged pixels. (2) False positive (FP) number: the number of pixels that did not change were finally detected as changed pixels. (3) True positive (TP) number: The number of pixel changes detected correctly. (4) True negative (TN) number: the number of pixels that are correctly detected without change. (5) Total error pixels (Over Error, OE) number: represents the sum of the number of missed detections and the number of false detections, and its expression is: $O E=F P+F N$. (6) Percentage Correct Classification (PCC): its expression as:

$$
P C C=\frac{T P+T N}{T P+F P+T N+F N}
$$


(7) Kappa coefficient:

$$
\text { Kappa }=\frac{P C C-P R E}{1-P R E}
$$

where $P R E$ is expressed as follows:

$$
P R E=\frac{(T P+F P) \cdot N_{c}+(T N+F N) \cdot N_{u}}{\left(N_{c}+N_{u}\right)^{2}}
$$

The Kappa coefficient represents the similarity between the detection result and the reference image. The larger the value, the closer to the reference image and the better the detection effect. In formula 3-3, $N_{c}$ and $N_{u}$ respectively represent the changed number and unchanged number of the actual detected pixels.

\section{EXPERIMENT RESULT}

\subsection{Experimental Data Set}

The first set of SAR image data was taken by Radarstat Remote Sensing in June 1997 and August 1997, respectively, which showed the surface change caused by the flooding in Ottawa, Canada during the rainy season and the Ottawa data set for short, as shown in Figure 4-1. (a) and Figure 3-1(b). The size of the data set is $290 \times 350$, and Figure $4-1$ (c) represents the reference image of actual changes in the surface.

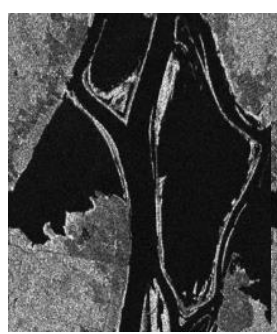

(a)June, 1997

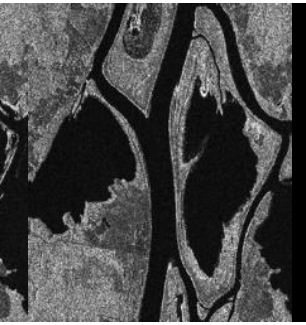

(b) August, 1997

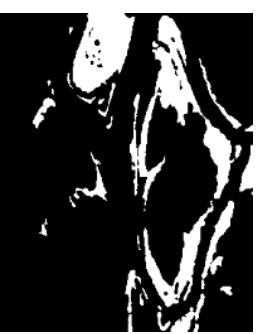

(c) reference
Figure. 4-1 Ottawa dataset

The second data set is the changes in the area of the Yellow River estuary. It is the remote sensing images taken by the Radarsat-2 satellite on June 18, 2008 and June 19, 2009, respectively. The size of the data set is $289 \times 257$ pixels. Figure 4-2(c) reflects the reference image of actual changes on the surface.

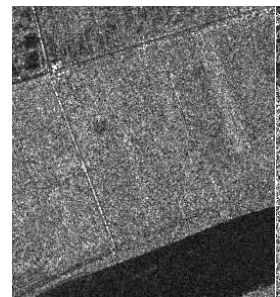

(a) June 18, 2008

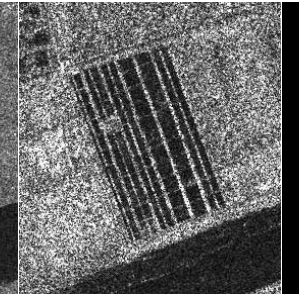

(b) June 19, 2009

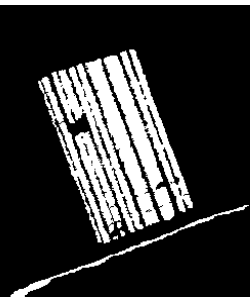

(c) reference

\subsection{Analysis of Experimental Results}

The hardware environment of experiment is Intel Core(TM) i5 CPU, main screen $1.8 \mathrm{GHZ}$, memory 8GB. The software environment is Matlab R2016b. In order to verify that the multi-scale feature extraction proposed in this paper can generate high-performance difference image, enhance image contrast and enhance edge contours of change regions, in this section, the logarithmic ratio difference image, the neighborhood ratio difference image, and the multi-scale feature extraction difference image proposed in this paper and

the gray histogram corresponding to each difference image are compared and analyzed. The relevant parameter settings in the experiment include: the local window size of the pooling weight kernel matrix $k=3$, the fuzzy factor $m=2$, the number of cluster centers $c=2$, the termination iteration condition $\varepsilon=10^{-5}$, and the number of pixel neighborhoods $N_{R}=9$. Regarding the setting of cumulative weighting times $\mathrm{T}$, it will be discussed and analyzed at the end of this section.

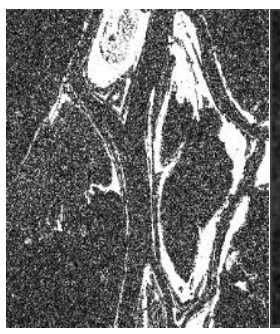

(a) LR image

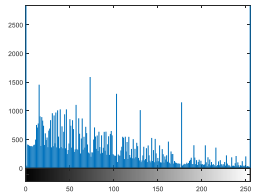

(d) LR histogram

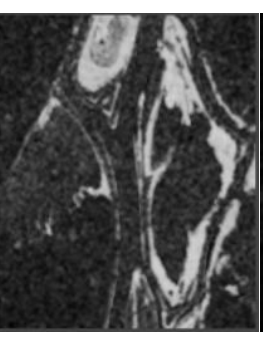

(b) NR image

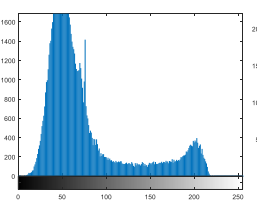

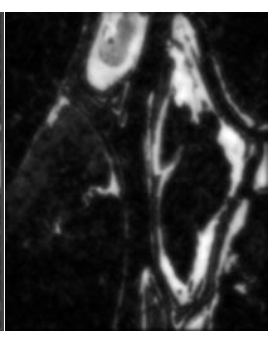

(c) proposed

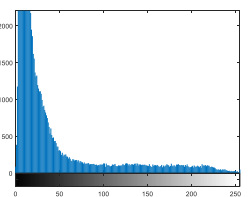

(f) Multi-scale feature

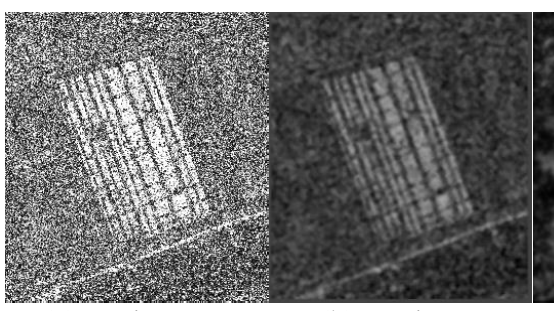

(a) LR image

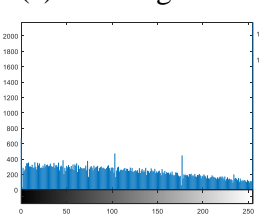

(d) LR histogram (b) NR image

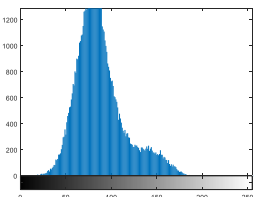

(e) NR histogram

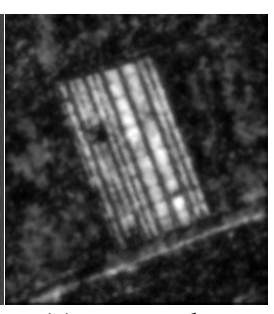

(c) proposed

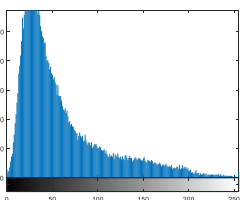

(f) Proposed
Figure. 4-4 difference image and histogram of Yellow River dataset

It can be seen from the difference image and the gray-scale histogram that the pixel distribution of the image in the LR method is relatively uniform, and the brightness of the image as a whole is also relatively uniform. The brightness of the changed area is higher than the brightness of the NR method, but there is more speckle noise. The pixels of the NR method are mainly distributed in the middle gray area, and the background of the difference image is relatively dark, which appropriately suppresses the background information, but blurs the changing area of the image. However, the pixels of the multi-scale feature histogram are mainly distributed in low grayscale areas, and the grayscale value range of the grayscale histogram is also wider, indicating that the contrast of the image is higher, which can also indicate that the method can effectively suppress the image background information to enhance the contrast between the changed area and the unchanged area. 
4.2.1 Experimental results and analysis of the Ottawa data set

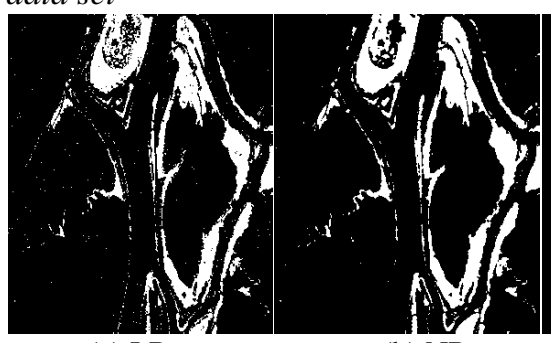

(a) LR (b) NR

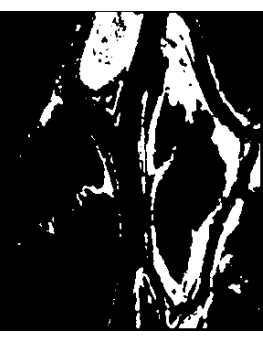

(c) proposed
Figure. 4-5 Different difference operator detection results of Ottawa dataset

Table. 4-1 Detection results of different differential operators in Ottawa

\begin{tabular}{cccccc}
\hline algorithm & FP & FN & OE & PCC & Kappa \\
\hline LR & 3184 & 1258 & 4442 & $95.62 \%$ & $85.05 \%$ \\
NR & 1261 & 912 & 2173 & $97.86 \%$ & $91.58 \%$ \\
Proposed & 925 & 860 & 1785 & $98.24 \%$ & $93.41 \%$ \\
\hline
\end{tabular}

Table 4-1 and Figure 4-5 are the change detection results of the Ottawa area under three different difference operators. It can be seen from Fig. 4-5 that there are many false positive and false negative pixels in Fig. 4-5(a). The logarithmic ratio method has a weak anti-noise ability, which leads to a larger total number of errors for the entire pixel. Figure 4-5(b) Compared with 4-5(a), the number of false detections is greatly reduced due to the effective using of the neighboring information of the pixels, but there are still many false detection areas in the overall image, and the edge contours of the changed areas are not clear. Figure 4-5(c) has reduced the number of false detections, which is reflected in the reduction of false detection areas at the edge of the changing area, so the total number of errors is also appropriately reduced.

\subsubsection{Experimental results and analysis of the Yellow}

\section{River basin dataset}

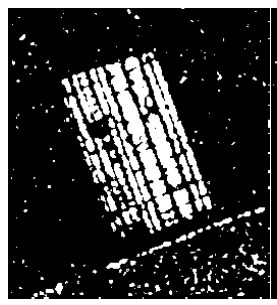

(a) LR

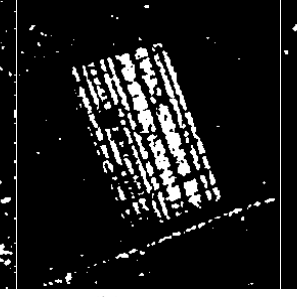

(b) NR

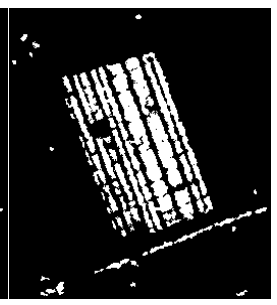

(c) proposed
Figure. 4-6 Different difference operator detection results of Yellow River basin dataset

Table. 4-2 Detection results of different differential operators in Yellow River basin

\begin{tabular}{cccccc}
\hline algorithm & FP & FN & OE & PCC & Kappa \\
\hline LR & 2937 & 2408 & 5387 & 92.75 & 74.81 \\
NR & 675 & 3748 & 4423 & 94.04 & 77.93 \\
Proposed & 928 & 2325 & 3253 & 95.62 & $85.56 \%$ \\
\hline
\end{tabular}

Table 4-2 and Figure 4-6 show the change detection results under three different difference operators in the Yellow River Basin. As can be seen from Figure 4-6, Figures 4-6(a) and 4-6(b) have more pseudo-change information, but they are reflected in different aspects. Figure 4-6 (a) shows a large number of missed detections and false detections, so there are more white spots in the image. Figure 4-6(b) There are a large number of missed detection areas inside the change area, which may be caused by the low contrast of the difference image, but because the neighborhood ratio method uses the neighborhood factor of the pixel, there are fewer false detection pixels. Figure 4-6(c), the number of missed detections in the relative neighborhood ratio method is greatly reduced. This is because the difference operator enhances the change area of the image, and the relative log ratio method effectively reduces the interference of speckle noise and the change area The edge detail information remains intact.

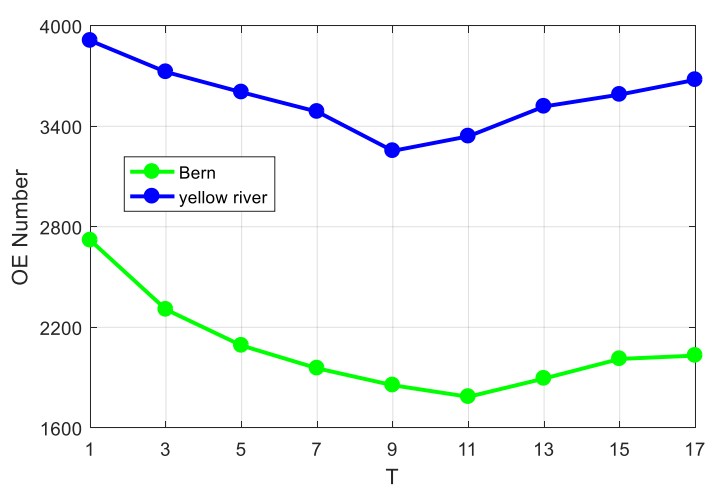

Figure. 4-7 Relationship between the OE values and T

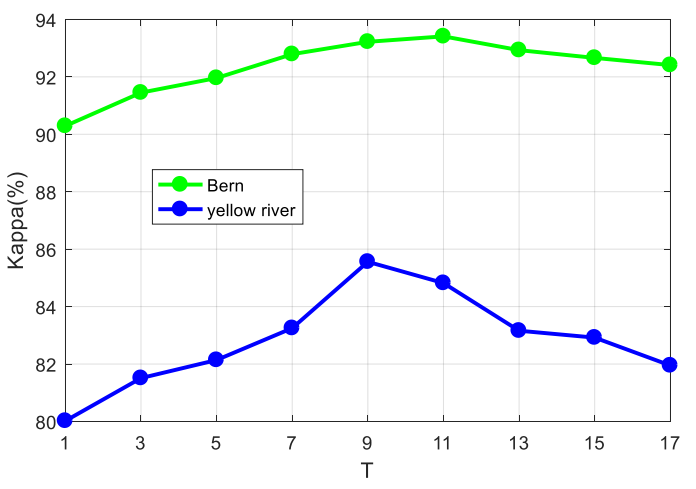

Figure. 4-8 Relationship between the PCC values and T

Figure 4-7 and Figure 4-8 discuss the influence of the cumulative weighted pooling times $T$ on the Kappa coefficient and the total number of error pixels OE. As can be seen from the figure, the impact on different data set parameters is different. For the Yellow River Basin, when T is 9, the Kappa coefficient reaches its maximum. Through the above analysis, it can be found that for the Ottawa data set, because the pixels in the change area account for a large proportion, the number of times the feature needs to be extracted is large.

\section{CONCLUSION}

This paper proposes a SAR image change detection algorithm based on multi-scale feature extraction, which improves the accuracy of detection and the similarity between the image and the reference change map. In this paper, the weighted pooling kernel matrix of different window sizes can be used to extract features of different scales of the image, which can enhance the contrast of the difference image. Then the cumulative weighted average of the pixel values of different window sizes can further reduce the sparse high-value noise 
on the logarithmic ratio image, deeply filter the speckle noise in the image, and suppress the background information of the image. In addition, this paper combines the FCM_S clustering algorithm to extract the change area. Experiments prove that the difference image generated by the difference operator in this paper is visually superior to the traditional logarithmic ratio operator and neighborhood ratio operator. At the same time, it can be seen that the detection accuracy of the algorithm in this paper has been improved to a certain extent through the detection data, which verifies the effectiveness and feasibility of the algorithm in this paper. However, the adjustment coefficient control algorithm has certain limitations on the robustness of noise, and there is still room for improvement in the accuracy of the algorithm as a whole.

\section{REFERENCES}

[1] Wang R., Song J. 2008. SAR image classification based on improved FCM algorithm. Journal of Northwest University.

[2] Zhao Z, Jiao L, Hou B., et al. 2016. Locality-constraint discriminant feature learning for high-resolution SAR image classification. Neurocomputing.

[3] Lary D. J., Alavi A. H., Gandomi A. H., et al. 2016. Machine learning in geosciences and remote sensing. Geoscience Frontiers.

[4] Malmgren-Hansen D., Engholm R., Pedersen M. O. 2016. Training convolutional neural networks for translational invariance on SAR ATR. In Proceedings of EUSAR 2016: 11th European Conference on Synthetic Aperture Radar.

[5] Gao F., Dong J., Li B., et al. 2017. Automatic change detection in synthetic aperture radar images based on PCANet. IEEE Geoscience and Remote Sensing Letters.

[6] Hou B., Wei Q., Zheng Y., et al. 2014. Unsupervised change detection in SAR image based on gauss-log ratio image fusion and compressed projection. IEEE Journal of Selected Topics in Applied Earth Observations and Remote Sensing.
[7] Inglada J. and Mercier G. 2007. A new statistical similarity measure for change detection in multitemporal SAR images and its extension to multiscale change analysis. IEEE Transactions on Geoscience and Remote Sensing.

[8] Gong M., Cao Y. and Wu Q. 2012. A neighborhoodbased ratio approach for change detection in SAR images. IEEE Geoscience and Remote Sensing Letters.

[9] Gong M., Zhou Z. and Ma J. 2012. Change detection in synthetic aperture radar images based on image fusion and fuzzy clustering. IEEE Transactions on Image Processing.

[10] Jiang H., Yang X., Dong Z., et al. 2017. Change detection for remote sensing images based on combined difference image. Journal of Hefei University of Technology.

[11] Zhou W., Jia Z., Yang J., et al. 2018. Change detection in SAR images based on combined different image and FCM clustering.

[12] Zhang X., Su H., Zhang C., et al. 2020. A robust imbalanced SAR image change detection approach based on deep difference image and PCANet.

[13] Shang R., Xie K., Okoth M. A., et al. 2019. SAR image change detection based on mean shift pre-classification and fuzzy C-means. In Proceedings of 2019 IEEE International Geoscience and Remote Sensing Symposium.

[14] Ahmed M. N., Yamany S. M., Mohamed N., et al. 2002. A modified fuzzy C-means algorithm for bias field estimation and segmentation of MRI data. IEEE Transactions on Medical Imaging.

[15] Lu D., Mausel P., Eduardo B., et al. 2004. Change Detection Techniques. International Journal of Remote Sensing. 